\title{
Emission Line Properties of Seyfert 1 Type AGN from RASS
}

\author{
D. W. Xu ${ }^{1,2}$, S. Komossa ${ }^{1}$, J. Y. Wei ${ }^{2}$, X. Z. Zheng ${ }^{2}$, and Y. Qian ${ }^{3}$ \\ ${ }^{1}$ Max-Plank-Institut für extraterrestrische Physik, Giessenbachstrasse, \\ D-85748 Garching, Germany \\ ${ }^{2}$ National Astronomical Observatories, Beijing 100012, China \\ ${ }^{3}$ Tsinghua University, Beijing 100080, China
}

\begin{abstract}
We present the optical emission line properties of a sample of 155 bright X-ray selected ROSAT Seyfert 1 type AGN. The measured properties are gathered for correlation analysis. The strong correlations between $\mathrm{H} \beta$ redshift, flux ratios of Fe II to $\mathrm{H} \beta$ broad component and [O III] to $\mathrm{H} \beta$ narrow component are found.
\end{abstract}

\section{Introduction}

Optical spectra of Active Galactic Nuclei (AGN) exhibit an extremely wide variety of properties. Multi-wavelength observations of well-defined source samples have proved a effective means in understanding the physical processes in AGN. A large and homogeneous X-ray selected AGN sample is therefore in great importance in studying the correlations between the optical to soft X-ray continuum and optical emission line properties of AGN.

The present sample of 155 Type 1 AGN results from the the optical identification of the X-ray sources with high X-ray to optical flux ratio $\left(f_{X} / f_{o p t}\right)$ discovered in the RASS (Wei et al. 1999). Forty out of 155 sources are known AGN which meet our selection criteria. $^{1}$

\section{Optical Measurements}

In many of the spectra there is a clear contribution from blends of Fe II line emission on both the blue and red sides of the $\mathrm{H} \beta-[\mathrm{O}$ III] complex. In order to reliably measure line parameters and to determine the strength of the Fe II emission, we have carefully removed the Fe II multiplets following the method described by Boroson \& Green(1992) which relies on an Fe II template.

\footnotetext{
${ }^{1}$ The selection criteria of the sample are: an alternative high X-ray-to-optical flux ratio criterion, i.e., $\log C R \geq-0.4 R+4.9$, where $C R$ and $R$ represent $X$-ray count rate and $R$ magnitude respectively; declination $\delta \geq 3^{\circ}$; galactic latitude $|\mathrm{b}| \geq 20^{\circ}$; optical counterparts within a circle with radius $\mathrm{r}=\mathrm{r}_{1}+5^{\prime \prime}$, where $r_{1}$ is the RASS position error given by Voges et al.(1996); optical counterparts with $\mathrm{R}$ magnitudes between 13.5 and 16.5 .
} 
The Fe II subtracted spectra were used to measure the non-Fe II line properties. We have assumed that the emission line profiles can be represented by a single or a combination of Gaussian profiles.

The optical index $\alpha_{\text {opt }}$ was calculated using the continuum flux density at $4400 \AA$ and $7000 \AA$ in the rest frame. The X-ray spectral slope $\alpha_{x}$ is defined between 0.1 and $2.0 \mathrm{keV}$.

\section{Correlations Analysis}

we explore whether the various emission-line and continuum properties correlate with one another. For this purpose, we calculated the the Spearman rank-order correlation matrix, along with its significance matrix for measured properties. A set of 12 different properties results in 66 correlation coefficients. Among them, 31 correlations were found with two-sided probabilities Ps $<0.01$.

The most striking correlations found in the present work are three correlations involving the $\mathrm{H} \beta$ redshift (or blueshift), flux ratios of $\mathrm{Fe}$ II to $\mathrm{H} \beta$ broad component (Fe II $/ \mathrm{H} \beta_{b}$ ) and [O III $]$ to $\mathrm{H} \beta$ narrow component ([O III $\left.] / \mathrm{H} \beta_{n}\right)$. The correlations covering $[\mathrm{O}$ III $] / \mathrm{H} \beta_{n}$ have never been investigated by other authors so far. There is a trend that strong Fe II $/ \mathrm{H} \beta_{b}$-weak $[\mathrm{O}$ III $] / \mathrm{H} \beta_{n}$ objects tend to have blueshift in $\mathrm{H} \beta$ while strong [O III] $/ \mathrm{H} \beta_{n}$ - weak $\mathrm{Fe} \mathrm{II} / \mathrm{H} \beta_{b}$ tend to have redshift in $\mathrm{H} \beta$. These strong correlations between $\mathrm{Fe} \mathrm{II} / \mathrm{H} \beta_{b}, \mathrm{H} \beta$ redshift and [O III] $/ \mathrm{H} \beta_{n}$ must reflect some physical connection between broad and narrow line emitting regions. Our results reinforce the interpretation of the "Eigenvector 1 correlations" found in Boroson \& Green (1992) as driven mainly by $L / L_{E d d}$ (Boroson 2002).

Acknowledgments. This work was partly supported by NSFC under grant 19973014. DX acknowledges a MPG-CAS exchange program.

\section{References}

Boroson, T.A. 2002, ApJ, 565, 78

Boroson, T.A., \& Green R.F. 1992, ApJS, 80, 109

Voges, W., Aschenbach, B., Boller, Th., et al. 1996, IAU Circ. 6420

Wei, J.Y., Xu, D.W., Dong, X.Y., \& Hu, J.Y. 1999, A\&AS, 139, 575 